\title{
Design of an Agent-Based Model to Simulate Governance in Inter- Organizational Project Networks
}

\author{
J. Kujala ${ }^{1}$, T. Vuorinen ${ }^{1}$ \\ ${ }^{1}$ Indusrial Engineering and Management, Faculty of Technology, University of Oulu, Finland \\ (jaakko.kujala@oulu.fi)
}

\begin{abstract}
Agent-based simulation models provide a means of analyzing and understanding the functioning of complex socio-economic systems, which often involve multiple interacting entities and non-linear and stochastic interactions. In such models, agents may be individuals or organizations, characterized by such properties as objectives, values, capabilities and resources. Project networks are temporary inter-organizational networks of heterogeneous actors whose purpose is to accomplish a complex set of tasks and reach shared goals. The variety of actors in project networks and the complexity of their interactions makes agent-based simulation a logical choice for simulating such networks. This paper present a design of an agent-based simulation for simulating governance in project network. Key design principles are introduced, along with practical examples of how specific governance mechanisms can be implemented in the model. Additionally, advantages and challenges of agent-based simulation as a research method is discussed.
\end{abstract}

Keywords - Agent-based simulation, governance, modeling, project networks

\section{INTRODUCTION}

Project networks are temporary inter-organizational networks of heterogeneous actors [1], [2]. These networks are task-oriented-that is, participating actors must accomplish a complex set of tasks or a project. Although they can to a certain degree be considered to share a goal and agreement on how to reach it, each actor may have their own goals, values and working methods, which complicate the control and coordination of the work. The challenge is all the greater as, by definition, no single organization in a network has full control over other actors. Instead, governance in the project network emerges in part from the structure of the network and the interactions among actors, as well as from the institutional context in which the project is embedded [3].

Governance in project network in this paper is defined as "the coordination, adaptation and safeguarding mechanisms that enable multiple organizational actors in project networks to work toward shared or joint goals" [3]. Governance itself is a broad concept, research to improve understanding of governance in project networks is challenging. Additionally, projects and the contexts in which they are implemented each have unique characteristics that influence the design and emergence of project network governance, making it difficult to apply or compare results across projects. Accessing empirical data that describe at a practical level what actors are doing and which factors they consider in actual decision making situations involves a multi-organizational setup. As a consequence, most of the research describing project network phenomena is fairly abstract and is based mainly on the perspective of specific actors. The complexity of these phenomena also makes it difficult to apply quantitative research methods. For these reasons, our understanding of governance in project networks remains limited, as research results are descriptive, context dependent, and often difficult to apply in practice.

Simulation is often presented as a third research method that may complement inductive and deductive reasoning based on empirical data [4]. The main objective of this paper is to introduce the design of agent-based modeling for simulating governance in project networks and discuss how it can be used to support research and managerial decision making.

The paper is structured as follows. We first discuss simulation as a research method. Second, we introduce previous work in the area and provide a brief discussion of the phenomenon, governance in project networks, to be simulated. Third, a design of a simulation model and its implementation is presented. Finally we discuss how the design of the simulation model introduced in this paper can provide a foundation for further development project simulation, and what are the potential research and practical contributions.

\section{SIMULATION AS RESEARCH METHOD}

Simulation can be understood as the process by which a model yields insight into the behavior of a "real system" [5]. Models do not attempt to imitate that real system but to capture a few key properties that provide an insight into its behavior. Three basic simulation paradigms (modelling methods) for computer simulation are discrete event or process simulation, system dynamics and agent-based simulation [6]. An agent-based simulation method was selected for this research as it provides the means for analyzing and understanding the functioning of complex socio-economic systems [7]. Comprising many interacting entities and non-linear interactions that are often stochastic in nature, the behavior of systems such as interorganizational project network cannot be effectively described using process models and system dynamic modelling cannot capture the heterogeneity and micro level behavior related to decision making of individual actors.

In agent-based models, agents interact with other agents and with an environment. Agents may be any type heterogeneous entities that are characterized by such properties as goals, decision making, competences and resources memory, and capability to adapt behavior [8] 
The behavior and actions of agents is described through decision rules; the multiple actions of each independent agent contribute to complex system behavior, and may also lead to unanticipated macro-behaviors of groups and networks of firms [9], [10]. In this model the main entities that are modelled as agents are projects, tasks, organizations and individuals working in organizations and making decisions.

\section{DESIGN OF THE SIMULATION MODEL}

\section{A. Previous research and theoretical foundations}

The design of the simulations systems builds on and continues the work of the Virtual Design Team (VDT) research group at Stanford University [11], [12]. That research was initiated at the Center for Integrated Facility Engineering (CIFE) in the late 1980s to help managers to design organizations and work processes for fast-track development of complex products without incurring the large cost overruns and catastrophic quality failures that frequently plagued such efforts. The VDT/Power simulation is based on an information-processing view of the organization [13]. The main focus in VDT/Power is on indirect work resulting from project-related communications and exceptions in a single organization setting, while the present model focuses on how governance mechanisms influence work practices in a multi-organizational setting. The governance mechanisms are assumed to influence both organizations motivation and capability to work towards common goals [3]. They include

- Goal setting to create performance goals that are shared by all project actors

- Rewarding to align actors' goals with project goals

- Monitoring to ensure that all actors behave as expected

- Coordination to align the behavior of actors to work effectively together

- Role definition and decision making to give power to make decision related to project implementation in appropriate level

- Capability building to ensure that project actors can meet performance expectations

While the governance literature is concerned mainly with the cost of controlling opportunistic behavior and less with the coordination of work [14], the complexity of the task in project contexts also highlights the importance of coordination. This may include such elements as shared information systems, role definition, decision making structures and competence requirements for each participating actor. It follows that project actors need to be both motivated and capable of working toward shared project goals. Figure 1 illustrates how governance mechanisms influence project actors' motivation and capability to act in a manner that ensures meeting project objectives.

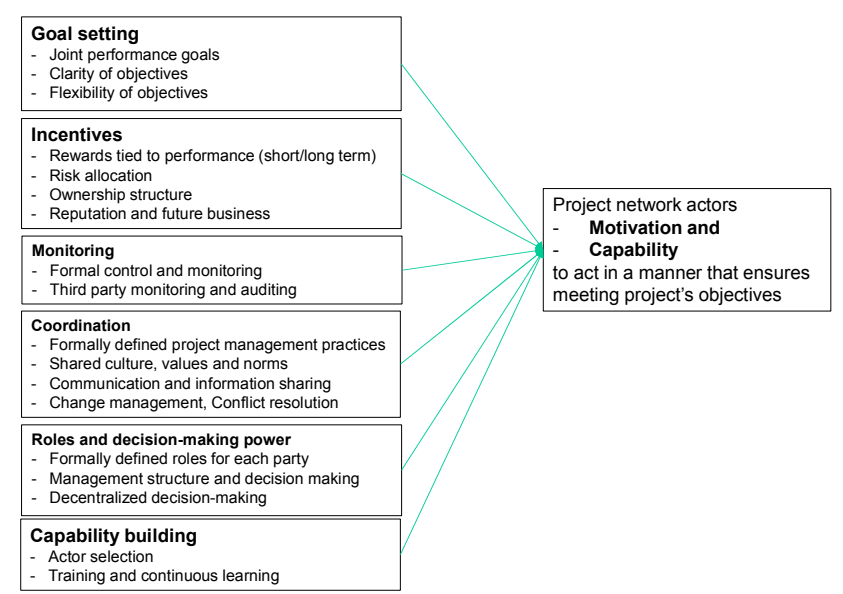

Fig. 1. : Governance mechanisms to facilitate willingness and ability to meet project performance objectives.

\section{B. Design of the simulation model}

The simulation comprises two modules: 1) a project module or task network that creates work and assigns work to actors and 2) a project network module that includes all actors and relationships between. The basic modules, and how they are related are illustrated in figure 2 .

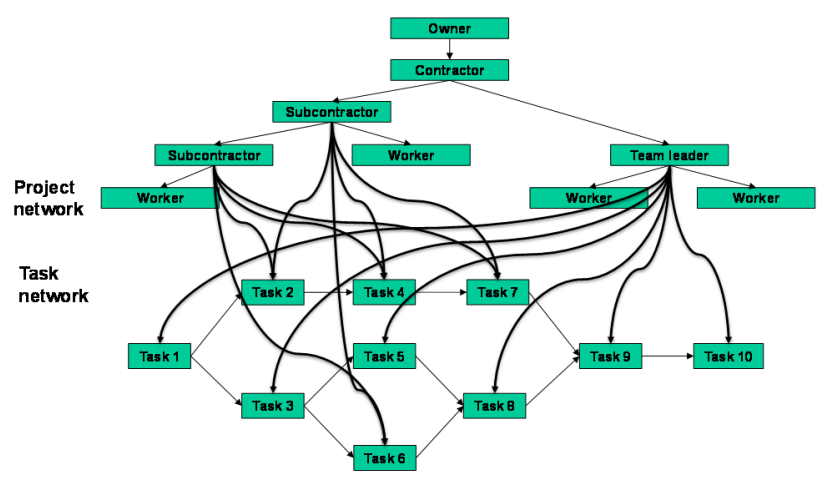

Fig. 2. Task network and inter-organizational project network

The project module creates projects and tasks in a specified order. Tasks are assigned to actors, who are responsible for completing that task. There is uncertainty related to task duration, and each task requires specific competences for its completion. Actor's skills influence how fast and what is the quality level of the work that will be accomplished. The extent of monitoring practices will influence whether low quality work will be noticed and rework is created. During the process actors learn and can do similar tasks faster and with better quality.

Each task may have several states. It will start after all preceding task in the task network are completed. While in progress or rework state it will create work packages for actors to work with, and when task is completed a request is sent to a manager to approve the work. Similarly, actors may be off-duty, idle or working. 
They may do project work, attend meetings, solve problems and make decisions. In figure 3 the simplified process overview is presented.

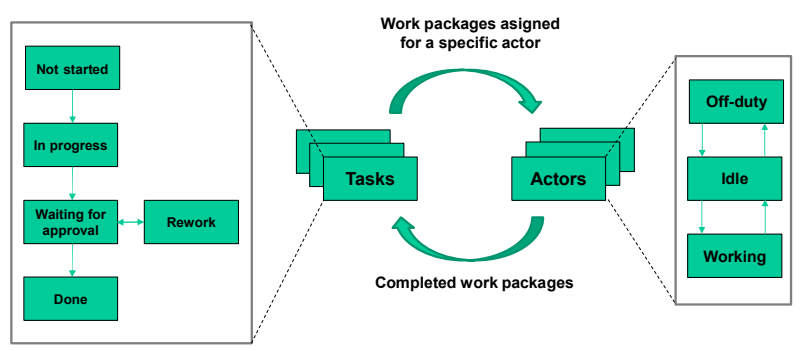

Fig. 3. Process overview

Governance mechanisms influence which task actor selects to work with if there are several competing tasks to be done. For example, in case compensation is based on delivering the project on-time, the actor can be assumed to focus on task that are on critical path and/or even work overtime to accomplish task in time. Governance mechanisms also influence how capable actors are in completing task in time and with high quality. For example, selection of actors based on their skills and not only based on lowest prices for difficult and time critical tasks is essential. Task are also interrelated having information links between them and may be processed in parallel, which requires frequent exchange of information between actors working in those tasks. This may be accomplished for example by having meeting to share information, which in turn takes time from actual work.

\section{Implementation of the simulation model}

The simulation model was implemented using Anylogic 7.3 (University edition). This software was selected because it provides a graphical interface for the design of the simulation that is relatively easy to use. Although based on the Java programming language, the interface hides some of the technical complexities of Java. As commercially developed software, it offers good support and includes a lot of learning material and example models. Anylogic also includes experiments such as parameter variation and sensitivity analysis to examine the effect of changing model parameters, which with are important features for practical use of the simulation model and for model validation [15].

The main objective for practical implementation of the model was to provide a foundation for the development of robust basic platform with easy-to-use interfaces, allowing features to be added or modified to address specific research problems or practical project design issues. All input data for the project can be imported from an Excel data file, making it easy to change the project design and to simulate various options.

\section{Verification and validation}

The main challenge in using simulation as a research method relates to the verification and validation of simulation models. Verification is the process of determining whether the simulation model as implemented (often created by software specialist) corresponds to the conceptual model created by the researcher. Fortunately, this is mainly a technical challenge, and there are various software engineering methods for overcoming this problem, including well designed documentation and programmatic testing of the model's code, test cases and scenarios [15]. Validation, on the other hand, refers to how well the implemented model corresponds to reality. The challenge here is that the available data on micro-level decision making and processes may be insufficient. Simulation is often also based on simplified assumptions, which makes it difficult to validate results using macro level empirical data. However, simulation can be valuable research tool even when grounding is not possible [7]. The process of designing simulation model and exploring consequence of theoretically derived micro-level process may lead to new insight and increase our understanding of the factors influencing project performance.

The model presented in this research brings up the main design principles and provides practical validation that this type of model is useful for simulating the effect of governance mechanisms for project performance. As such it provides a starting point for the further development of project simulation models, but this specific model has not been verified using systematic software engineering methods nor validated using robust empirical data.

\section{DISCUSSION}

\section{A. Research contribution of agent-based simulation}

The main objective for this research was to provide a basic design of an agent-based model, which could be used as a starting point for further research. From a research perspective, agent-based modeling of project design and implementation may provide additional insights into the processes and micro-level issues that influence project performance. Specifying agent-based models forces the researcher to focus attention on microlevel behavior, decision-making and relationships between actors, and on how different design choices-in this case, governance mechanisms-influence project performance. The advantage and at the same time challenge with agent-based simulation models is that they require researcher to be very explicit with their reasoning. As such they can be used to complement existing descriptive qualitative research on project networks, but the challenge is how to operationalize often very fuzzy theoretical concepts that are created as results of qualitative studies. 
One of the main challenges in project research is that all project are by definition unique and their uniqueness influences project implementation and the effectiveness of different management approaches. The collection of data from large sample of project, each having multiple partners, has proven to be very challenging. Simulation can be used to overcome this challenge as the cost of running multiple simulations with different parameters is cost efficient. It can be cost efficiently used to analyze of how different contingency variables influence project performance.

Additionally, collecting longitudinal data how complex project system develops over time from large sample of projects is not feasible. By simulating and collecting data on system behavior over time, project simulations can be used to create process theories [4], [17], which provide a better understanding of the underlying mechanisms and processes leading to project success.

\section{B. Simulation as a decision-making tool}

Simulation and modelling is often presented as method to create and explicate theories, but one of the objectives for modeling and simulation can also be to create realistic models that capture key features of real-world systems [7]. This type of models can be used as a tool to analyze impact of different organizational design choices and to support managerial decision making. It can provide a cost-effective means of testing which type of governance system would be most optimal for project performance and/or assessing current project performance based on projects' current technical and organizational design. In this role, simulation serves as a tool for managers "to design organizations as engineers design bridges" [11]. A key element in making simulation useful for project managers is the integration of simulation with project design and management tools and IT systems. This integration is especially relevant in the context of flexible approach to project design and implementation such as agile project management. Moving from a planningoriented approach to agile project management emphasizes the importance of being able to continuously analyze the impact of design changes and to respond to unexpected events during project implementation. In figure 4 the integration of simulation with management system is illustrated.
Design and implementation of a complex construction project

Project initial design, changes in the design and progress information

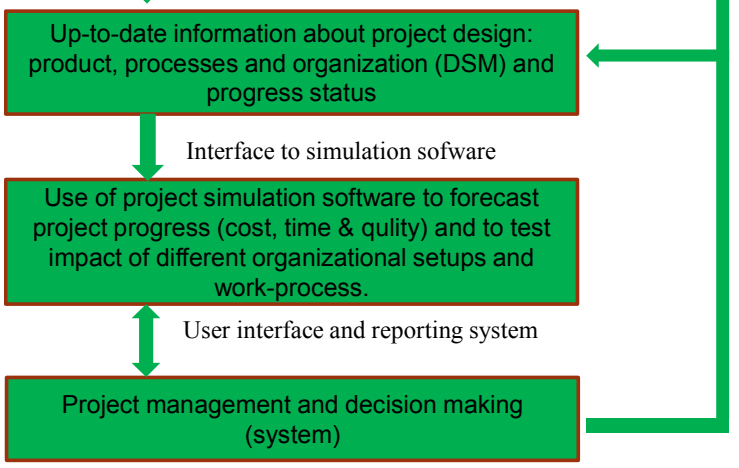

Fig. 4. Integration simulation with project management system

\section{Simulation as game or educational tool}

An agent-agent based simulation model can be extended and modified as a game by replacing some agents with human operators, allowing us to study decision making in complex situations and to create more realistic models. Additionally, this approach can be used to train people to understand a system's functioning and how their decisions may influence its effectiveness.

\section{CONCLUSION}

In this paper, simulation is presented as a method for studying governance in complex project networks. It is suggested that simulation can serve both as a tool to advance our theoretical understanding of project governance and to enhance decision making practices in project context. A description of a basic design of an agent-based project simulation model is provided. It can be used as a starting point to create model that can be used to analyze how different governance mechanisms influence a) overall efficiency in meeting project goal(s) and objectives; b) the distribution of value extracted from the project by different stakeholders; and c) how the project changes the network of participating companies.

Additionally, the project task network defines the key characteristics of project complexity: structural complexity (number of tasks and their interconnectedness) and uncertainty related to each task [17]. Thus it is also possible to modify these project characteristics and analyze how they moderate the efficiency of governance mechanisms from a contingency perspective. An example of such research would be how risk sharing and contract type influences profitability of the project for different actors with different levels of project complexity. 


\section{REFERENCES}

[1] T. Ahola, "Efficiency in Project Networks: The Role of Inter-Organizational Relationships in Project Implementation". Doctoral dissertation, 2009, Aalto University, Espoo, Finland.

[2] K. Artto, J. Kujala. "Project business as a research field, International Journal of Managing Projects in Business, 2008, vol. 1, no. 4, pp. 469-497.

[3] J. Kujala, K. Aaltonen, N. Gotcheva, A. Pekuri. "Key dimensions of project network governance and implications for safety in nuclear industry projects". Paper presented at EURAM 2016 Annual Conference, 1-4 June 2016, Paris.

[4] K. Miller. "Agent-based modeling and organization studies: A critical realist perspective". Organization Studies, vol. 36, no. 2, pp. 175-196

[5] G. Fioretti. "Agent-based simulation models in organization science”. Organizational Research Methods, vol. 16 , no. 2, pp. 227-241.

[6] A. Borshchev, A. Filippov. "From system dynamics and discrete event to practical agent based modeling: Reasons, techniques, tools". The 22nd International Conference of the System Dynamics Society, 2004, July 25-29, Oxford, England.

[7] R. Harrison, G. Carroll, K. Carley. "Simulation modeling in organizational research". Academy of Management Review, vo., 32, no. 4, pp. 1229-1245.

[8] C. Macal, M. North. "Agent-based modeling and simulation". Proceedings of the 2009 Winter Simulation conference.

[9] J. Epstein. "Agent-based computational model and generative social science”. Complexity, vol. 4, no. 5. Pp. 41-60.

[10] J. Sokolowski, C. Banks. "Modeling and Simulation Fundamentals: Theoretical Underpinnings and Practical Domains". 2010, Wiley.

[11] R. E. Levitt, J. Thomsen, T.R. Christiansen, J.C. Kunz, Y. Jin, C. Nass. "Simulating project work processes and organizations: Toward a micro-contingency theory of organizational design". Management Science, vol.4, no. 11, pp. 1479-1495.

[12] R.E. Levitt. "The virtual design team: Designing project organizations as engineers design bridges". Journal of Organization Design, vol. 1, no. 2, pp. 14-41.

[13] J.R. Galbraith. "Designing complex organizations". 1973, Addison-Wesley, Reading, MA.

[14] R. Gulati, P. Lawrence, P. Puranam. "Adaptation in vertical relationships: Beyond incentive conflict". Strategic Management Journal, vol. 26, pp. 415-440.

[15] W. Rand, R. Rust. "Agent-based modeling in marketing: Guidelines for rigor". International Journal for Research in Marketing, vol. 28, no.3, pp. 167-280.

[16] A. Langley. "Strategies for theorizing from process data". Academy of Management Review, 1999, 24(4): 691-710.

[17] T. Williams. Modelling complex projects. 1999, Wiley. 